\title{
Imaging Features of Postradiotherapy Changes in Head and Neck Cancers
}

\author{
Anisha Gehani $^{1}$ Saugata Sen ${ }^{1}$ Sanjoy Chatterjee ${ }^{2}$ Sumit Mukhopadhyay ${ }^{1}$ \\ ${ }^{1}$ Department of Radiology, Tata Medical Center, Rajarhat, Kolkata, \\ West Bengal, India \\ 2 Department of Radiation Oncology, Tata Medical Center, Rajarhat, \\ Kolkata, West Bengal, India \\ Address for correspondence Anisha Gehani, MD, Department of \\ Radiology, Tata Medical Center, Kolkata 700156, West Bengal, India \\ (e-mail: anisha.gehani@gmail.com). \\ Indian J Radiol Imaging 2021;31:661-669.
}

\begin{abstract}
Keywords

- postradiotherapy changes

- head and neck malignancy

- head and neck squamous cell carcinoma

- radiation complications

Radiation therapy is the mainstay in the treatment of head and neck cancers, in addition to surgery and chemotherapy. Expected radiotherapy changes evolving over time may be confused with recurrent tumor. Conversely, even residual or recurrent tumor in the setting of postradiotherapy changes may be difficult to identify clinically or even by radiological imaging. Therefore, it is important to be familiar with the temporal evolution of these changes. The purpose of this pictorial essay is thus to illustrate distinctly the expected radiotherapy changes and radiotherapy-related complications in the head and neck region and to differentiate them from tumor recurrence on routine cross-sectional imaging techniques (computed tomography and magnetic resonance imaging).
\end{abstract}

\section{Introduction}

With the diversity of tumors affecting the head and neck regions, radiotherapy (RT) protocols and their resultant changes are bound to vary. However, certain RT-related changes are inevitable. With the advent of newer RT techniques, such as three-dimensional conformal RT (3DCRT) and intensity-modulated RT (IMRT) replacing the conventional external beam RT (EBRT), the incidence of treatment-related complications have also reduced but it is still not negligible. ${ }^{1}$ Tumor per se, as well as surrounding soft tissues, cartilages, and bones in the treatment field can undergo various changes over time. Early complications related to mucositis are usually detected clinically and are beyond the scope of imaging. Late-term effects of RT may be confused with recurrent disease or with treatment-related complications and usually warrant imaging diagnosis.

DOI https://doi.org/ 10.1055/s-0041-1735921. ISSN 0971-3026.

\section{Approach to Posttreatment Neck}

The first step toward approaching a treated head and neck cancer scan is to review any previous imaging, including pretreatment scans. Adequate knowledge about type of surgery, including type of neck dissection and reconstructive flap used, is a must to be familiar with the expected changes. A knowledge of margins obtained at the time of surgery and primary histology of tumor by looking back at the surgical pathology report is just as important since some tumors have a high propensity for recurrence. Awareness of duration, dose, modality, location, and timing of RT is important. Finally, it is useful to know the reason for imaging, if there is any clinical suspicion of recurrence or only routine surveillance is required. Commonly used cross-sectional imaging modalities include computed tomography (CT), magnetic

\footnotetext{
(C) 2021. Indian Radiological Association. All rights reserved. This is an open access article published by Thieme under the terms of the Creative Commons Attribution-NonDerivative-NonCommercial-License, permitting copying and reproduction so long as the original work is given appropriate credit. Contents may not be used for commercial purposes, or adapted, remixed, transformed or built upon. (https://creativecommons.org/ licenses/by-nc-nd/4.0/) Thieme Medical and Scientific Publishers Pvt. Ltd., A-12, 2nd Floor,
} Sector 2, Noida-201301 UP, India 
Table 1 Overview of imaging modalities

\begin{tabular}{|l|l|l|}
\hline CT & MRI & FDG PET CT \\
\hline $\begin{array}{l}\text { Routinely used for all posttreatment } \\
\text { scans }\end{array}$ & $\begin{array}{l}\text { In the post treatment set up for select } \\
\text { cases of oral cavity, nasopharyngeal, } \\
\text { skull base, and sinonasal tumors }\end{array}$ & $\begin{array}{l}\text { Can be used in presence of contraindica- } \\
\text { tion to iodinated or gadolinium-based } \\
\text { contrasts }\end{array}$ \\
\hline $\begin{array}{l}\text { Rapid acquisition, good for patients } \\
\text { with swallowing, lying flat and } \\
\text { breath holding difficulty }\end{array}$ & Excellent soft tissue resolution & $\begin{array}{l}\text { Excellent modality for detecting locore- } \\
\text { gional recurrence and distant metastases } \\
\text { Good for detection of second primary } \\
\text { cancers }\end{array}$ \\
\hline $\begin{array}{l}\text { Better bone evaluation (osteora- } \\
\text { dionecrosis, new bone destruction } \\
\text { in recurrence) }\end{array}$ & $\begin{array}{l}\text { Good for evaluation of perineural tu- } \\
\text { mor spread and dural invasion }\end{array}$ & $\begin{array}{l}\text { Good for detection of primary site in case } \\
\text { of biopsy proven carcinoma of unknown } \\
\text { primary }\end{array}$ \\
\hline $\begin{array}{l}\text { Better evaluation of gas bubbles } \\
\text { around the cartilage } \\
\text { (chondronecrosis) }\end{array}$ & $\begin{array}{l}\text { Multiparametric MRI (diffusion, perfu- } \\
\text { sion, and contrast enhanced MRI) for } \\
\text { evaluation of tumor recurrence } \\
\text { Can also be used when dental amal- } \\
\text { gams interfere with CT interpretation }\end{array}$ & $\begin{array}{l}\text { Effectively identifies primary tumor site } \\
\text { when artifacts from dental amalgams may } \\
\text { interfere with image interpretation in CT } \\
\text { and MRI }\end{array}$ \\
\hline
\end{tabular}

Abbreviations: $\mathrm{CT}$, computed tomography; FDG PET, fluorodeoxyglucose positron emission tomography; MRI, magnetic resonance imaging

resonance imaging (MRI) and fluorodeoxyglucose positron emission tomography (FDG PET)-CT (-Table 1). In most cases, CT is complementary to MRI to evaluate osseous involvement and laryngeal invasions.

\section{Routine Surveillance}

Currently, no clear consensus exists among institutions regarding the optimum time of imaging or type of imaging modality to be routinely employed. The current National Comprehensive Cancer Network (NCCN) guidelines recommend baseline cross-sectional imaging at 8 to 10 weeks after treatment completion. But there are no recommendations for subsequent follow-up scan timings. If the specific clinical question is to look for residual or recurrent disease, following surgery or chemo-RT, the choice for baseline imaging is usually a contrast-enhanced CT scan of the neck and thorax or PET-CT study. Hence, in the surveillance setting too, there may be institutionalized protocols in place for time and type of imaging. In the author's institution, clinical examination and surveillance imaging are performed quarterly for the first 2 years, semiannually until 4 years and annually thereafter in the absence of a clinical suspicion.

This pictorial review aims to describe the early and late, expected, and unexpected changes of RT on standard CT and MRI examinations.

\section{Expected Postradiation Changes: Early}

These changes are expected to be seen within 3 months of treatment, and sometimes as soon as 2 weeks of treatment initiation and are mostly attributed to interstitial edema of the soft tissues due to disruption of endothelial cell lining, affecting venous and lymphatic drainage. ${ }^{2}$ These present as progressive thickening of skin and platysma (-Fig. 1), subcutaneous reticulations (-Fig. 2), and mucosal edema of pharyngeal and laryngeal walls. Sometimes, laryngeal edema can be very severe and life-threatening which should be

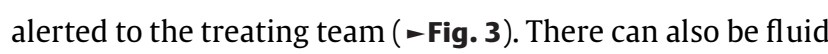
collections and edema of the retropharyngeal space which should not be mistaken for infectious processes ( - Fig. 4) as infective fluid collections are expected to have enhancing walls. Often, early radiation injury can involve the parotid and submandibular glands in a symmetric fashion which presents as diffuse enlargement and enhancement of the gland due to radiation-induced glandular hyperemia (-Fig. 5). Sometimes, these changes manifest asymmetrically involving only one gland, depending on the radiation portal causing a painless enlargement of only one gland (-Fig. 6). If superadded infection occurs due to poor oral hygiene, patients present with diffuse enlargement of the affected gland with pain and fever, termed as acute sialadenitis. Another expected change of RT includes fatty

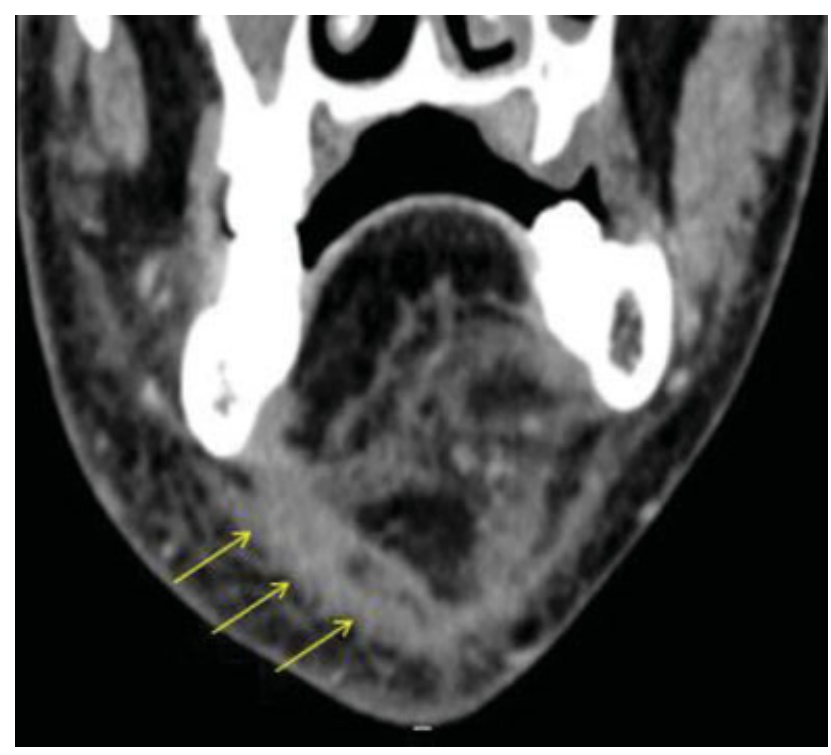

Fig. 1 A 49-year-old man operated for squamous cell carcinoma of the tongue. Coronal contrast-enhanced $\mathrm{CT}$ image shows progressive thickening of skin and platysma, asymmetrically more on the right (yellow arrows). CT, computed tomography. 


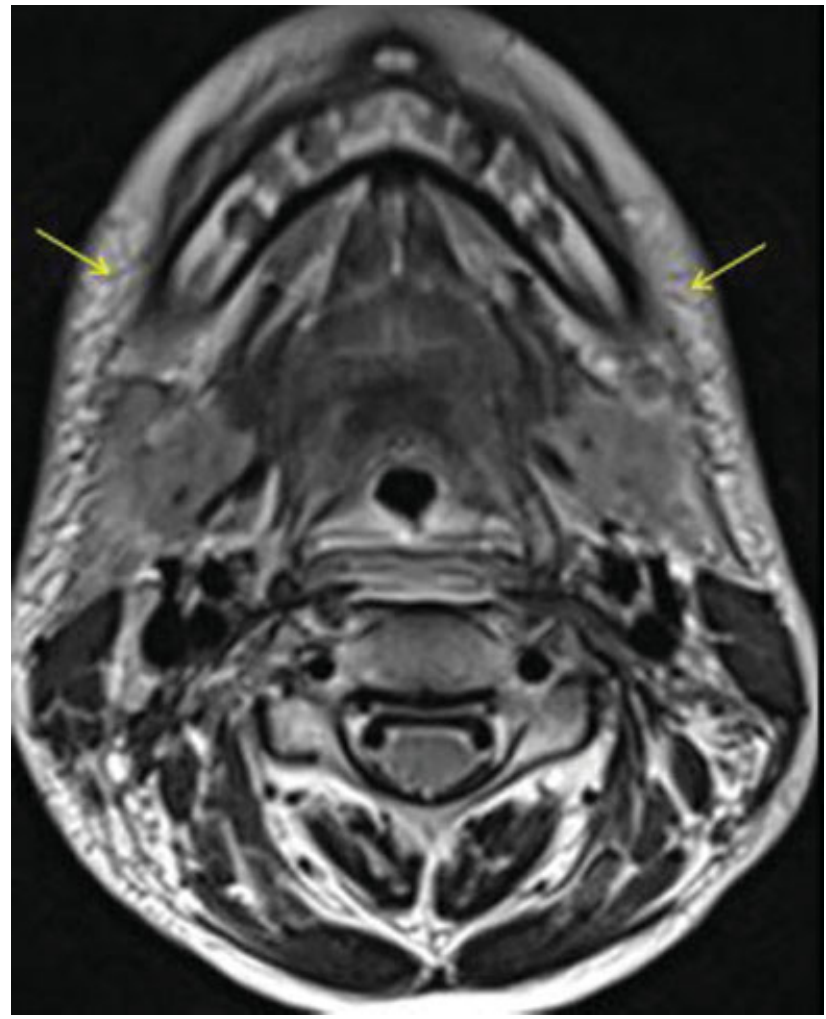

Fig. 2 A 12-year-old girl with nasopharyngeal cancer. Axial T2weighted MR image shows diffuse bilateral symmetrical reticulations in the subcutaneous fat (yellow arrows). MR, magnetic resonance.

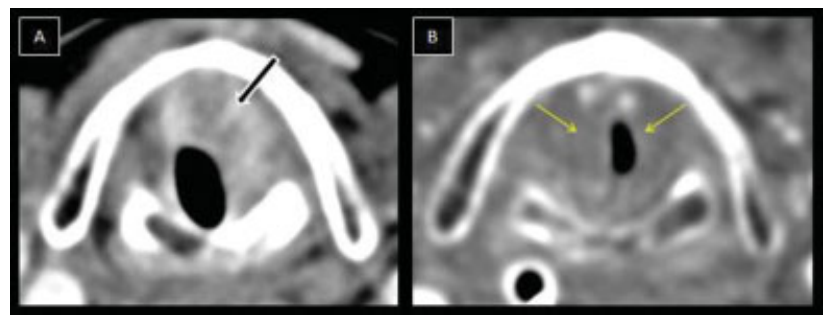

Fig. 3 A 55-year-old man with left vocal cord cancer. Axial contrastenhanced CT image prior to radiotherapy. (A) A mass (black arrow) in the left true cord and anterior commissure. Postradiotherapy axial contrast-enhanced CT image. (B) Extensive laryngeal edema (yellow arrows) causing narrowing of the airway and near complete resolution of the mass. CT, computed tomography.

conversion of bone marrow and is one of the most common osseous alterations seen as a result of RT ( - Fig. 7).

\section{Expected Postradiation Changes: Late}

These changes occur more than 3 months after RT completion and can persist for months to years and sometimes even resolve. Overtime, fibrosis and atrophy develop within the affected soft tissues lying in the radiation portal. ${ }^{3}$ The salivary glands and thyroid undergo a similar process, eventually undergoing atrophy and being replaced by fat.

Key point: expected tissue changes after RT appear symmetrical, unless the neck is irradiated using asymmetric radiation portals. Enhancement is homogenous without

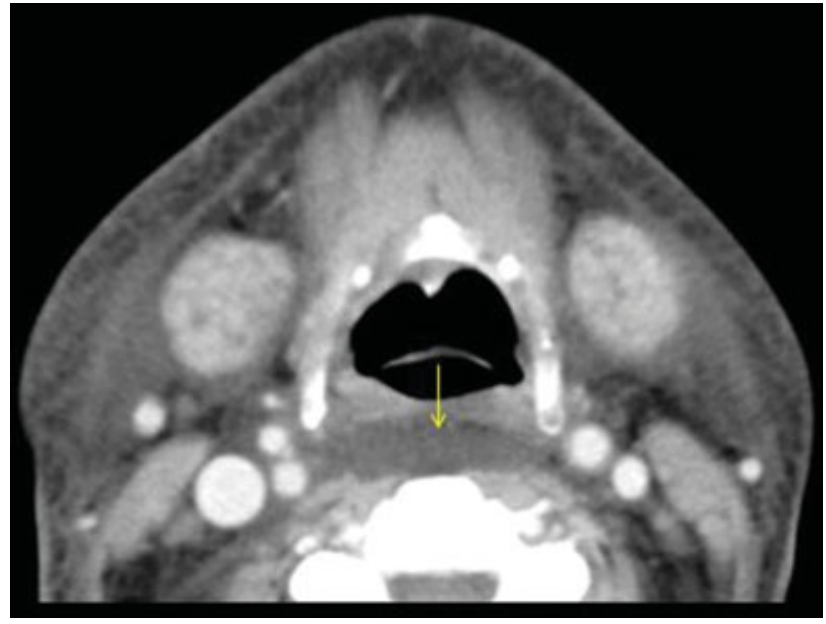

Fig. 4 A 61-year-old man with carcinoma base of the tongue. Axial contrast-enhanced CT image shows fluid collection and edema of the retropharyngeal space (yellow arrow). CT, computed tomography.

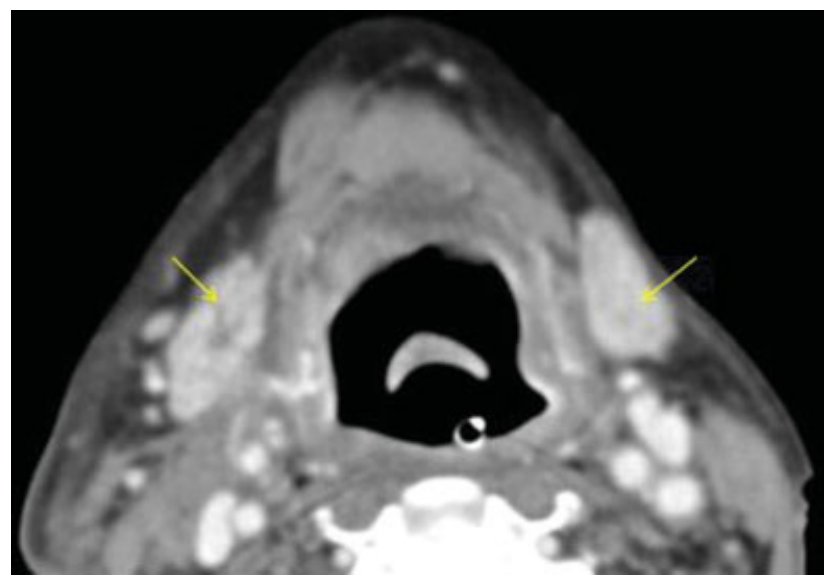

Fig. 5 A 59-year-old woman with carcinoma left buccal mucosa. Axial contrast-enhanced CT image shows hyper enhancing submandibular glands (yellow arrows) due to radiation-induced glandular hyperemia. $\mathrm{CT}$, computed tomography.

asymmetric expansion of affected soft tissues which helps differentiating from tumor recurrence.

\section{Unexpected Changes of Radiation Therapy}

The risk factors for complications of RT can be multifactorial and may be related to radiation dose, duration and fractionation, radiation field size, local extent and histology of the neoplasm, concurrent use of chemotherapy, continued consumption of tobacco and alcohol, dental infections, and dental extractions. ${ }^{1}$ These changes are seen for many months to years after therapy and generally follow a timeline (-Table 2).

\section{Radiation-Induced Lung Changes}

The lung apices are included in radiation treatment for head and neck malignancy to cover supraclavicular and level-IV cervical lymph nodes which result in bilateral apical radiation-induced changes. ${ }^{4}$ These changes manifest as acute 


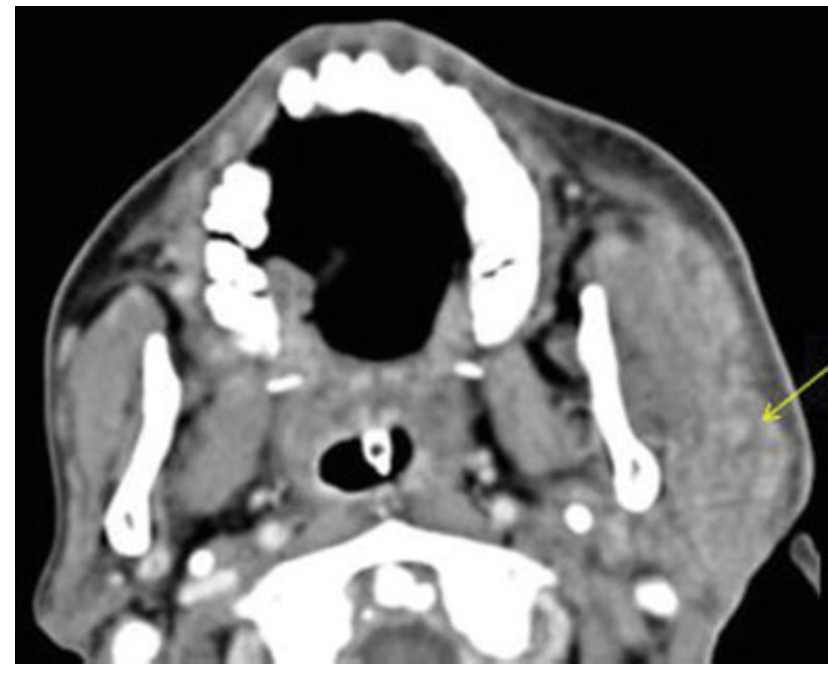

Fig. 6 A 58-year-old man with carcinoma left lower alveolus. Axial contrast-enhanced $\mathrm{CT}$ image shows asymmetric enlargement and enhancement of the left parotid gland (yellow arrow) due to an asymmetric targeted left radiation portal. CT, computed tomography.

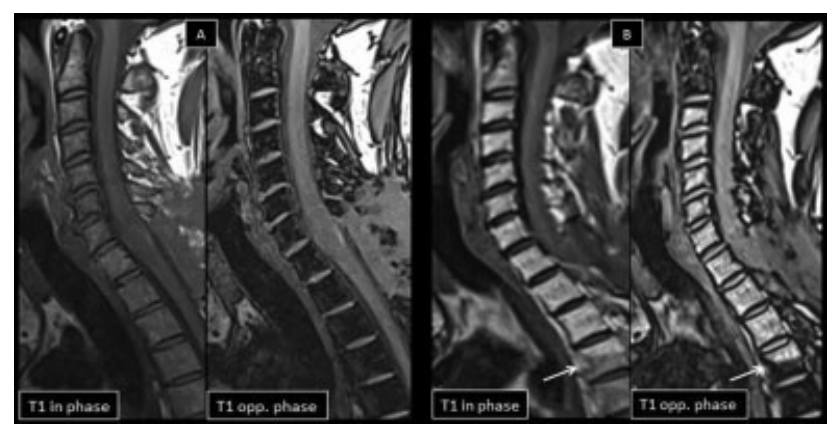

Fig. 7 A 48-year-old man with oropharyngeal carcinoma. (A) Sagittal T1-weighted in phase and opposed phase images show normal appearance of cervicodorsal spine marrow prior to radiotherapy. Note the conversion to fatty marrow from C3 vertebra to D4 vertebra with sharp margins corresponding with the radiation portal (white arrow) in the postradiotherapy in and opposed phase T1-weighted sagittal images (B).

Table 2 Expected timeline of radiotherapy complications

\begin{tabular}{|l|l|}
\hline Radiation-induced lung changes & Within 3-12 months \\
\hline Osteoradionecrosis & Within 12-18 months \\
\hline Chondronecrosis & Within 12-18 months \\
\hline Tumor recurrence & Within 18-36 months \\
\hline Radiation-induced brain changes & Within 24 months \\
\hline Radiation-induced vascular changes & $>$ 5-year latency period \\
\hline Radiation-induced neoplasias & $>5$-year latency period \\
\hline
\end{tabular}

radiation pneumonitis (within 1-3 months of therapy) or late radiation lung fibrosis (within 6-12 months after therapy). Imaging findings of radiation pneumonitis are focal ground-glass attenuation or consolidations or both (-Fig. 8). Radiation pneumonitis gradually resolves but it may progress to lung fibrosis if the damage is severe. Radia-

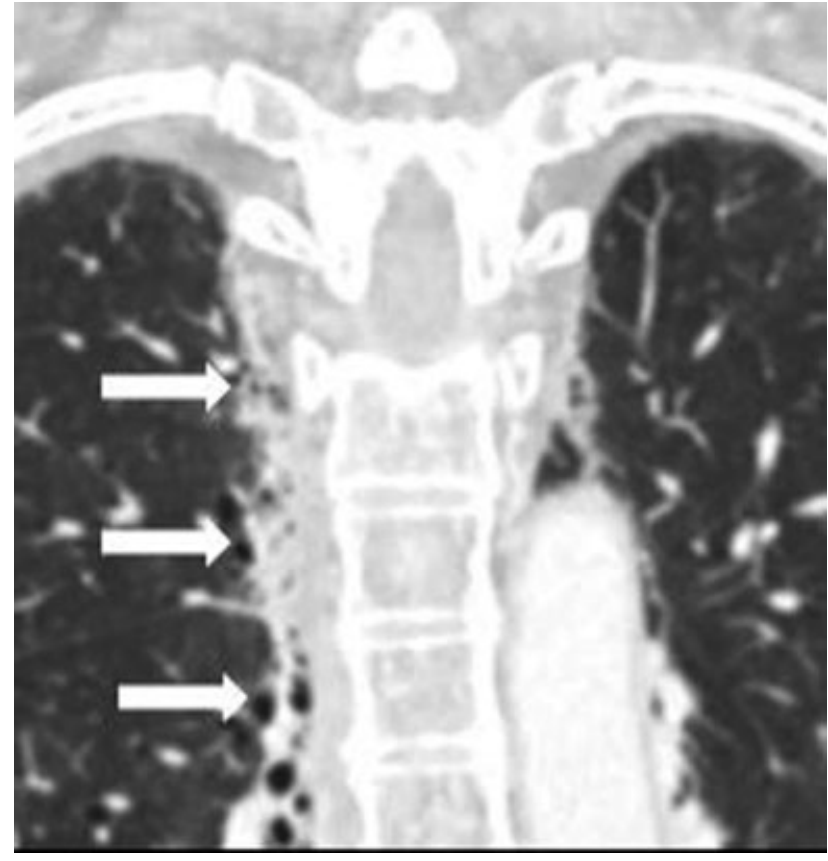

Fig. 8 A 64-year-old man with postcricoid carcinoma. Coronal reformatted CT image in lung window algorithm shows fibrosis of the lung due to chronic radiation pneumonitis (white arrows). CT, computed tomography.

tion lung fibrosis is seen on CT as a sharply delineated area of volume loss, linear scarring, and traction bronchiectasis.

\section{Osteoradionecrosis}

It is the necrosis occurring in the bone within an irradiated field, with formation of fistula on the overlying skin and persistent nonhealing for more than 3 months when radiation doses exceed $60 \mathrm{~Gy}$. At such high doses, osteoblastic function is impaired, resulting in decreased matrix production and increased bone resorption. Reparative processes ensue resulting in a mottled appearance with areas of patchy lucency, disorganized sclerosis, and coarsened trabeculae with or without bony expansion. ${ }^{5}$ Over time, there can be progressive necrosis, secondary infection, and bony sequestration. The risk is highest in the first 6 to 12 months after therapy but may persist years after RT. Commonly affected bones include the mandible (due to its unique blood supply and superficial location), maxilla, hyoid, skull base, and temporal bone. ${ }^{1,6}$ Osteonecrosis of the skull base is a very serious complication of RT and is rather rare presently due to the optimized organ sparing radiation techniques. We have not seen any cases of radio necrosis of the skull base at the author's institution over the past years.

CT shows bizarre lysis, disorganized sclerosis, and cortical destruction with or without clear fistula onto the skin surface and with disorganized sclerosis being the most common finding. MRI shows the affected bone with mixed altered signal intensity and irregular patchy enhancement. Some reports in literature have described the use of FDG PET-CT in diagnosing and differentiating osteoradionecrosis from tumor recurrence, but significant overlap exists in the standardized uptake value (SUV) values and the two cannot 


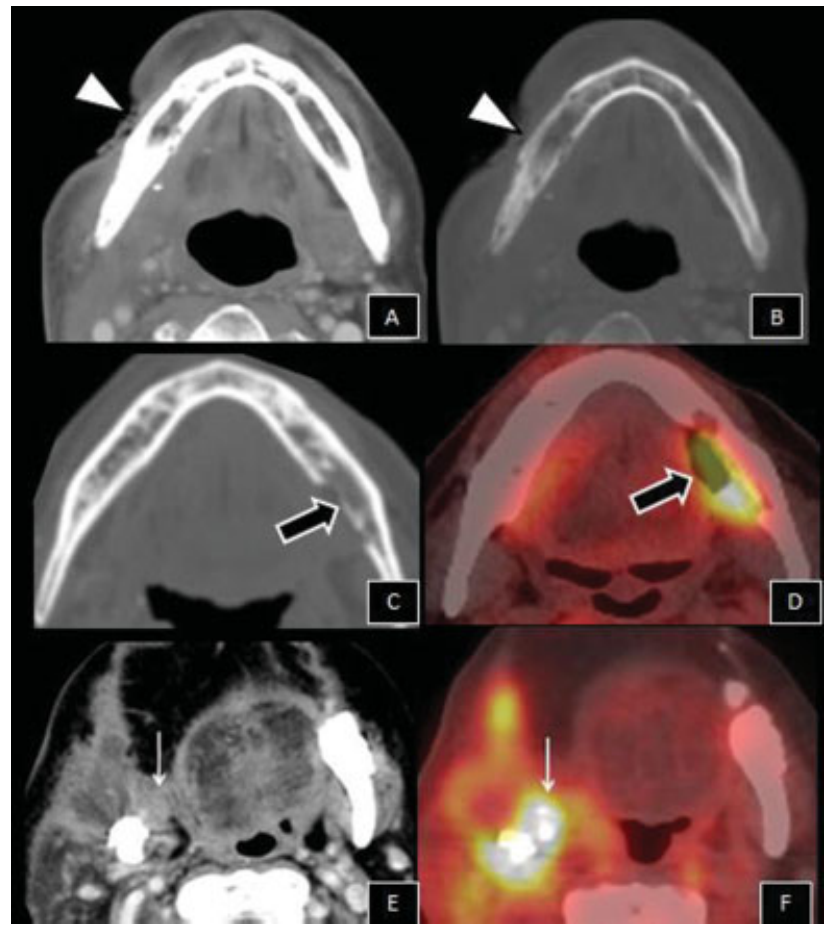

Fig. 9 Osteoradionecrosis versus tumor recurrence. Axial contrast-enhanced CT images in soft tissue window (A) and bone window (B) in a 66year-old man with carcinoma base of the tongue show cutaneous ulceration and bizarre lysis and sclerosis of right hemimandible respectively (white arrowheads) suggestive of osteoradionecrosis. In a different patient, axial $\mathrm{CT}$ image in bone window. (C) Lysis of the left hemimandible (thick black arrows) with corresponding uptake on FDG PET-CT image (D). Absence of expansile soft tissue at the site of bone destruction in these cases suggest osteoradionecrosis and thus ruling out possibility of recurrence. (E) Tumor recurrence shows focal enhancing mass (white arrow in E) corresponding to the site of bone destruction and FDG avidity (F). CT, computed tomography; FDG PET, fluorodeoxyglucose positron emission tomography.

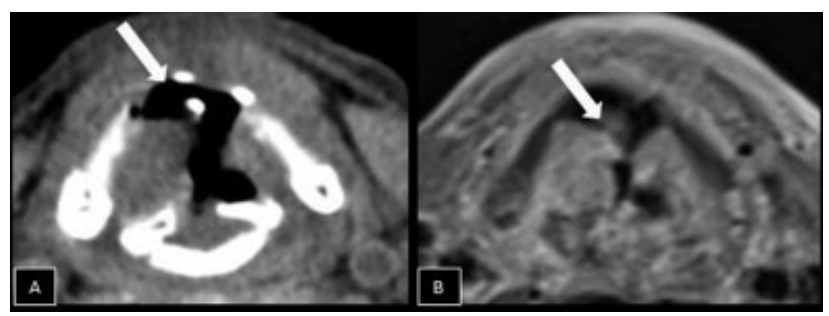

Fig. 10 Chondronecrosis. A 60-year-old man with carcinoma left vocal cord. Axial noncontrast CT image (A) and postcontrast T1-weighted fat saturated axial MR image. (B) Thyroid cartilage lysis (thick white arrows) with air foci in the vicinity and heterogeneous enhancement on MRI. CT, computed tomography; MRI, magnetic resonance imaging.

be differentiated without the help of key CT features as described. Key differentiating points of osteoradionecrosis from tumor recurrence are the presence of associated measurable enhancing soft tissue mass corresponding to the site of new bone erosion or lysis in the latter (-Fig. 9).

\section{Chondronecrosis}

It is the collapse of cartilage within an irradiated field due to breach in the perichondrium by tumor and superadded

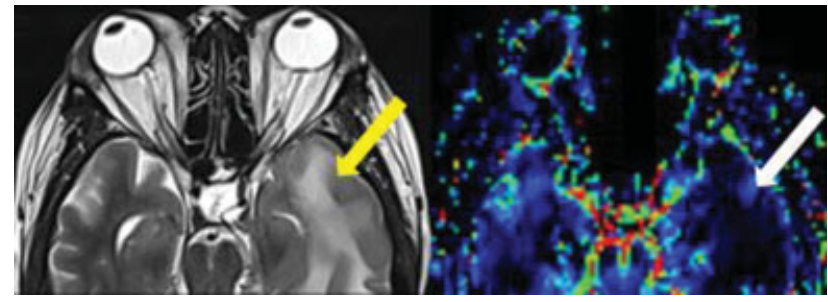

Fig. 11 Radiation-induced cerebral necrosis. A 32-year-old man with carcinoma left maxillary sinus. (A) Axial T2-weighted image and (B) corresponding perfusion maps show areas of white matter hyper intensity (yellow arrow) in the left temporal lobe and decreased CBV (white arrow) respectively suggestive of focal radiation-induced necrosis. CBV, cerebral blood volume.

infection resulting in necrosis. Late effects of radiation result in endothelial damage which impairs the vascular and lymphatic flow. Inflammatory changes ensue resulting in necrosis of the larynx and fistula formation with the overlying skin. Clinically, patients present with new onset odynophagia and hoarseness of voice. CT and MRI show fragmentation and collapse of affected cartilage ( - Fig. 10). Loss of high signal in the medullary space of ossified laryngeal cartilage on T1weighted images, and enhancement of the affected cartilage after injection of gadolinium-contrast agents is observed. ${ }^{7}$ Key differentiating features of chondronecrosis from tumor recurrence are presence of gas bubbles adjacent to the affected site of chondronecrosis, whereas presence of enhancing soft tissue clinches the diagnosis of tumor recurrence. Nevertheless, a coexistent tumor recurrence may be difficult to exclude depending on underlying tissue distortions.

\section{Cerebral Radiation Necrosis}

Radiation-induced brain necrosis occurs with a latency period of at least 2 years. It was more commonly associated with older RT techniques such as EBRT and would affect the medial and inferior temporal lobes when RT was used for the treatment of skull base or nasopharyngeal cancers. Incidence has reduced significantly with newer techniques like IMRT as the brain is spared and contoured out of the radiation field. ${ }^{8}$ It presents as a ring-enhancing lesion with perilesional edema involving the white matter on CT and MRI (-Fig. 11). Oftentimes, there may only be white matter T2 hyperintensity due to myelin dysfunction and gliosis in the absence of any enhancement. ${ }^{9,10}$ Differentials, including high-grade glioma, metastases, and infections, can be differentiated on the basis of combination of diffusion, MR perfusion, and MR spectroscopy.

\section{Radiation-Induced Herpes Simplex Encephalitis}

Although a rare phenomenon, herpes simplex encephalitis (HSE) in patients who have received RT for nasopharyngeal cancers has been reported in literature. It is postulated that cancer per se and related treatments, such as chemotherapy, RT, and corticosteroid, predispose to an immunosuppressive 


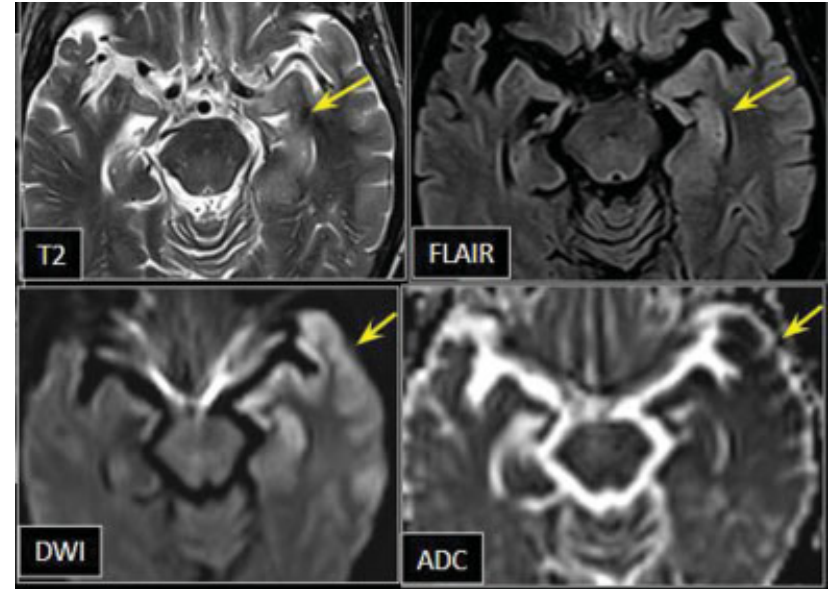

Fig. 12 Herpes simplex encephalitis. A 70-year-old man with nasopharyngeal cancer. Presented to the emergency room with acute onset confusion and altered behavior. MRI shows T2 and FLAIR hyperintensity and restricted diffusion in the left medial and lateral temporal lobes (yellow arrow). ADC, apparent diffusion coefficient; CSF VIROLOGY detected HSV1 DNA. CSF, cerebrospinal fluid; DWI, diffusion-weighted imaging; FLAIR, fluid-attenuated inversion recovery; MRI, magnetic resonance imaging.

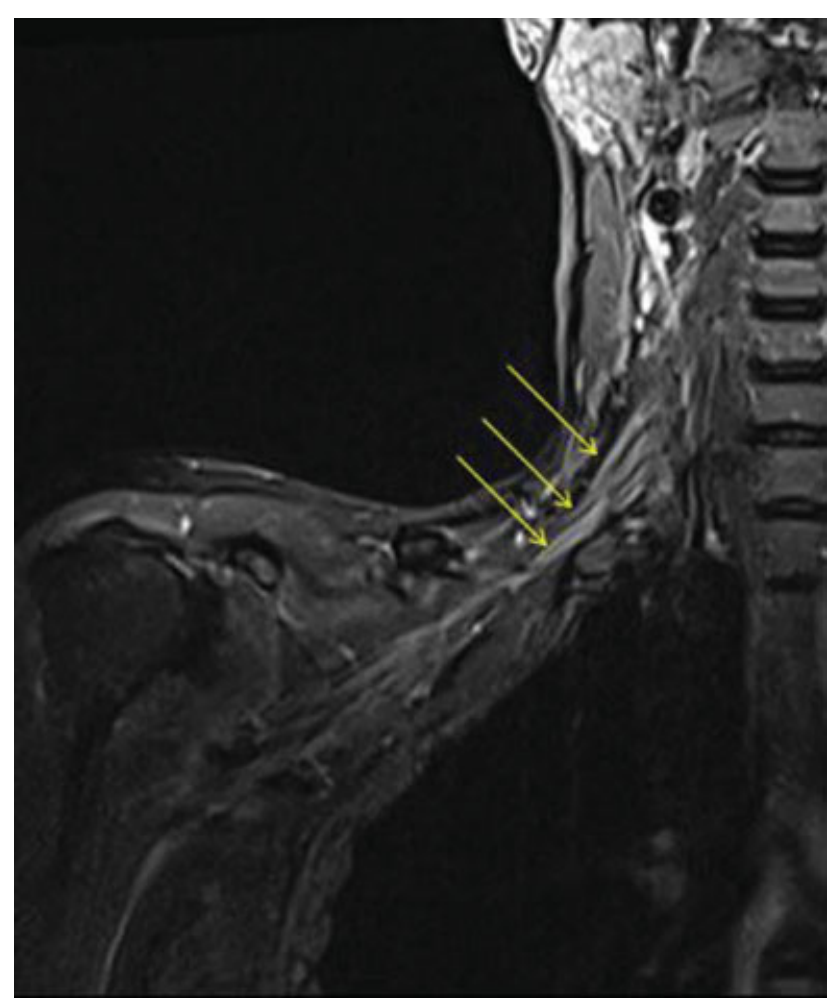

Fig. 13 Brachial plexopathy. STIR coronal image shows thickening and hyperintensity of trunks, divisions, and cords of the right brachial plexus (yellow arrows) in a 52-year-old man who received radiotherapy for oropharyngeal carcinoma. STIR, short-tau inversion imaging.

state resulting in an increased susceptibility to HSE due to disruption of blood brain barrier. ${ }^{11}$ Early diagnosis of HSE is essential, as the prognosis is dependent on early treatment. MRI is more sensitive than CT, especially diffusion-weighted images or fluid-attenuated inversion recovery (FLAIR) images that can identify abnormal areas. ${ }^{12}$ Unilateral temporal lobe

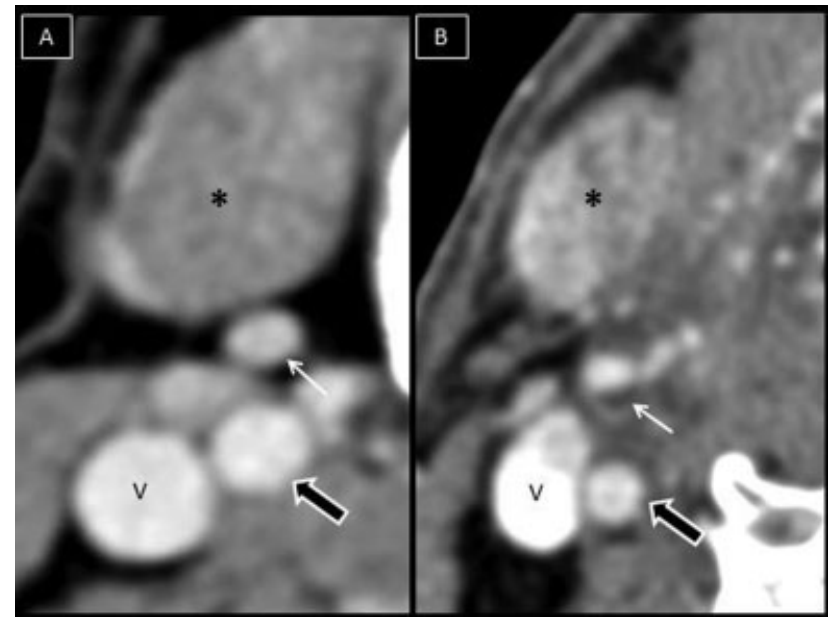

Fig. 14 Radiotherapy-related accelerated carotid artery atherosclerosis. (A) Axial contrast-enhanced CT image just above the level of right carotid artery bifurcation shows right external carotid artery (thin white arrow) and right internal carotid artery (thick black arrow) medial to the right internal jugular vein (V). (B) Postradiotherapy, axial contrast-enhanced $\mathrm{CT}$ image shows increased intimal thickness and fatty atherosclerotic plaque along the walls of right external carotid artery (thin white arrow) and right internal carotid artery (thick black arrow) causing mild luminal narrowing. Note the hyper enhancement as a result of radiotherapy in the right submandibular gland $\left({ }^{*}\right) . C T$, computed tomography.

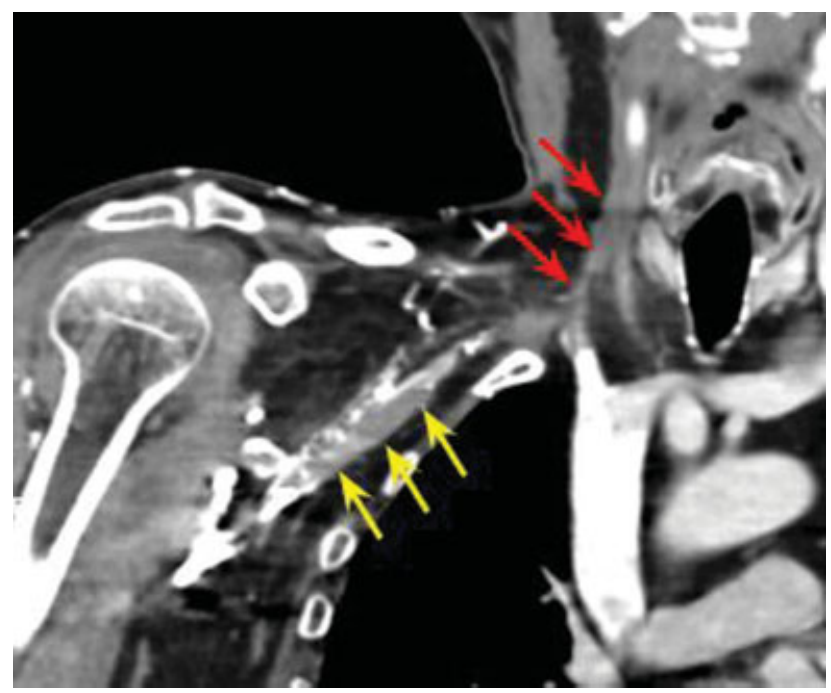

Fig. 15 Right internal jugular vein (IJV) and subclavian vein fibrosis in a 58-year-old man with carcinoma base of the tongue. Coronal reformatted contrast-enhanced $\mathrm{CT}$ image shows nonopacification right IJV (red arrows) due to narrowing and fibrosis of its lumen and partially fibrosed lumen of right subclavian vein (yellow arrow). CT, computed tomography.

and insular cortex involvement is the classic finding seen on imaging; however, bilateral involvement is also known to occur infrequently (-Fig. 12). Cerebrospinal fluid (CSF) polymerase chain reaction (PCR) has a sensitivity of $98 \%$ and specificity of 94 to $100 \%$, and is considered the gold standard for diagnosis. ${ }^{12}$. Early diagnosis and treatment remains the major prognostic indicator. 


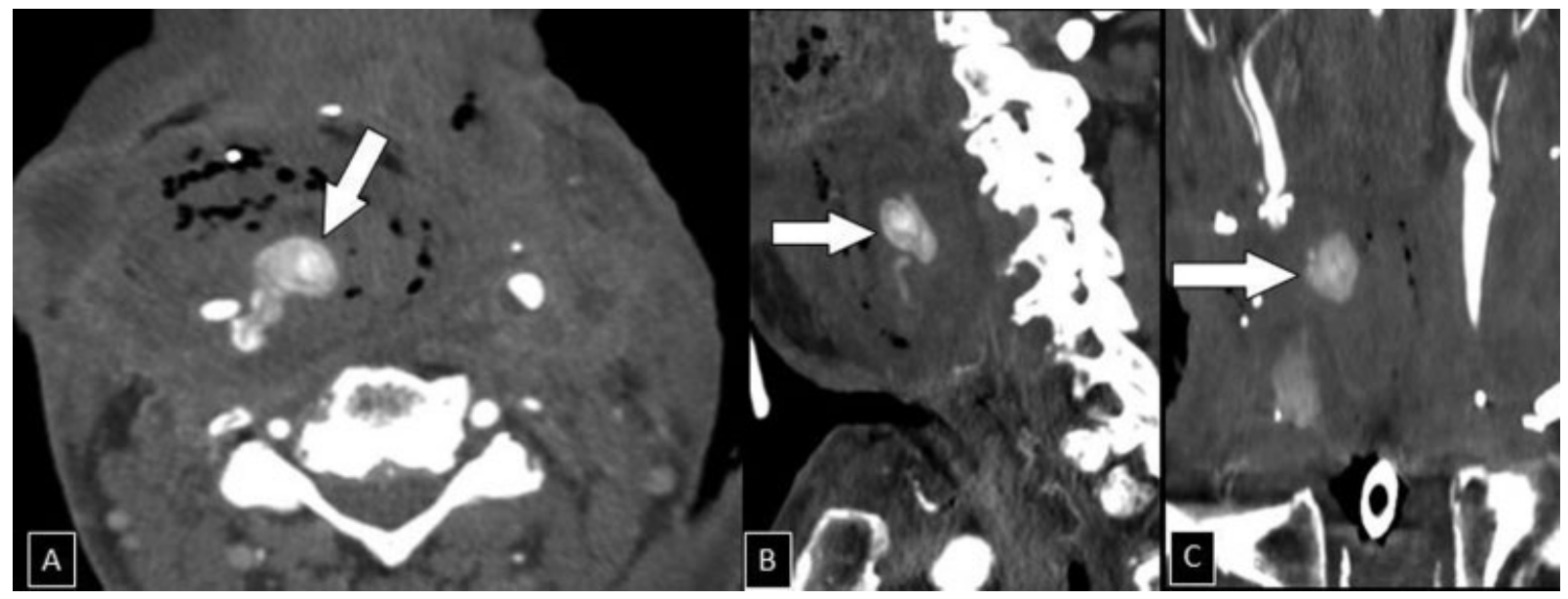

Fig. 16 Carotid artery pseudo-aneurysm. A 66-year-old woman with per-oral bleeding, 4 years after radiation therapy for right hypopharyngeal cancer. Contrast-enhanced CT images in axial (A), sagittal (B) and coronal (C) planes show right carotid artery pseudoaneurysm (thick white arrow) as the source of active bleed within the area of tumor recurrence. Case courtesy: Dr. Suresh Mukherji, MD. CT, computed tomography.
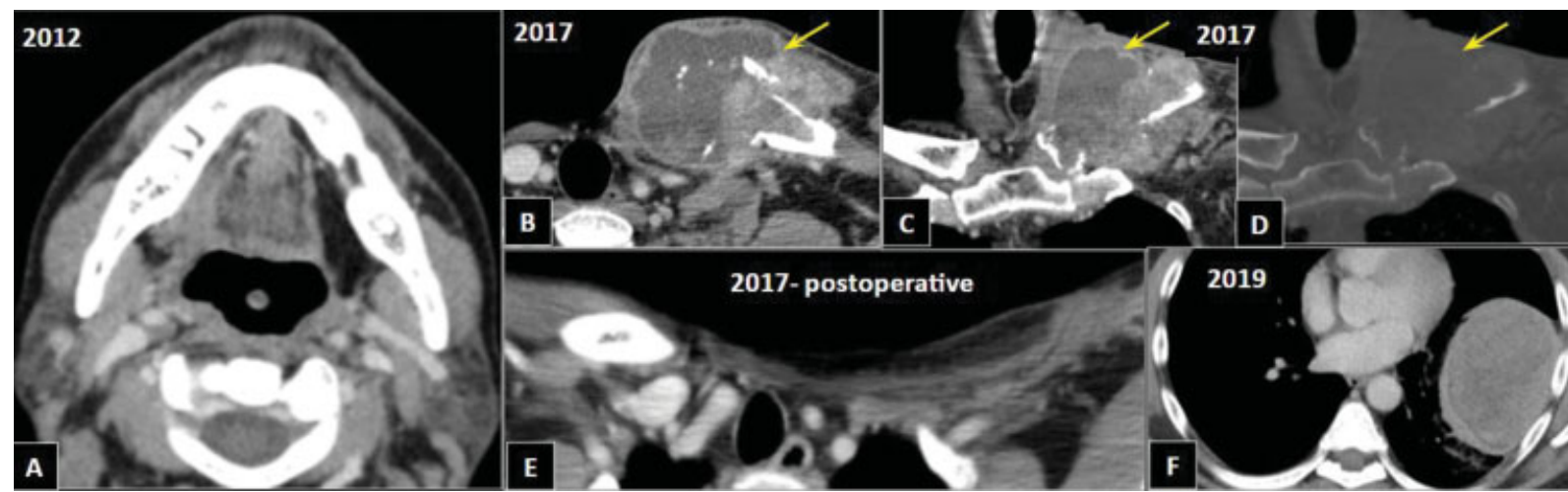
E
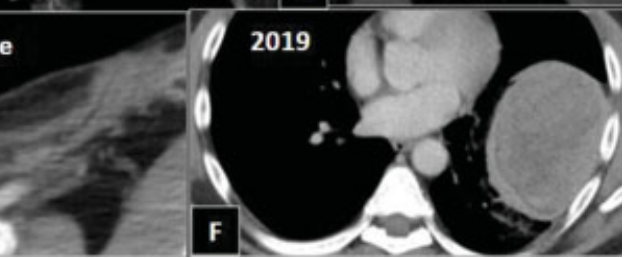

Fig. 17 Radiation-induced sarcoma. Axial contrast-enhanced CT image at the level of oral cavity (A) shows wide local excision of mass at left lateral tongue border in a 64-year-old man. Axial contrast-enhanced CT image (B) and coronal reformatted contrast-enhanced CT images in soft tissue window (C) and bone window (D) at the level of clavicle five years after treatment completion show an enhancing mass(yellow arrow) at the medial end of left clavicle with bone destruction, which was excised (E). Biopsy was high-grade pleomorphic sarcoma of left clavicle. Two years later, he developed a left lung mass (F). Biopsy was consistent with metastatic sarcoma. CT, computed tomography.

\section{Cranial and Brachial Neuropathy}

These changes occur along the same timeline as radiationinduced brain changes. Radiation-induced lower cranial nerve palsy may occur rarely and is thought to be due to fibrosis around the carotid sheath carrying cranial nerves 9 , 10,11 , and $12 .{ }^{13}$ Radiation-induced brachial plexopathy is also uncommon and peaks 2 to 4 years after treatment and can present as thickening and enhancement of affected roots and trunks (-Fig. 13). ${ }^{14}$

\section{Radiation-Induced Vascular Changes}

Large vessel vasculopathy is far less common than small vessel disease. It is thought to be a combination of radiation-induced intimal hyperplasia and accelerated atherosclerosis. The changes are seen only after high-dose therapy and with a variable latency period. Accelerated atherosclerosis (-Fig. 14), thrombosis of the internal jugular vein (-Fig. 15), arterial stenoses, and occlusions of the intracranial or extracra- nial circulation are the most common findings. ${ }^{15,16}$ These are also now becoming rare due to newer carotid sparing intensitymodulated RT techniques (CSIMRT). ${ }^{17,18}$ Pseudoaneurysms and carotid arterial blowouts are known but are rare complications that can result in profound and lethal bleeding perorally ${ }^{16}$ (-Fig. 16).

\section{New Primary Tumor and Radiation-Induced Neoplasms}

Positive association of head and neck squamous cell carcinomas with tobacco and alcohol consumption make these patients at an increased risk of a second primary neoplasm in the lungs or upper aerodigestive tract.

A radiation-induced secondary neoplasm is rare. It is imperative for the secondary tumor to be of a different histology than from the primary tumor and should occur at sites distant to the site of primary tumor and after a latency period of at least 5 years or more. ${ }^{1}$ Some common tumor histology include meningiomas, sarcoma, squamous cell 


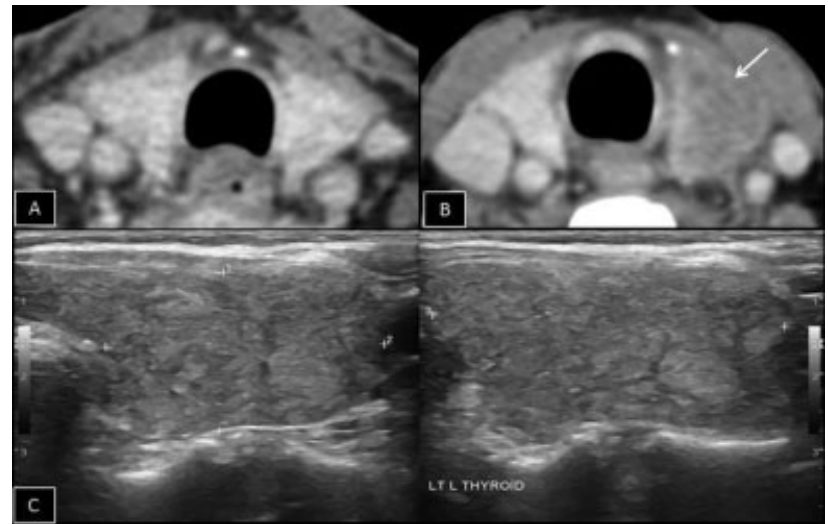

Fig. 18 (A-C) Post-radiotherapy thyroiditis. (A) Axial contrast-enhanced $\mathrm{CT}$ image at the level of the thyroid gland prior to radiotherapy shows normal thyroid gland size and attenuation. B. Axial contrastenhanced CT image at the level of the thyroid gland one year after radiotherapy for carcinoma larynx shows enlarged left lobe of the thyroid gland as compared to the right lobe with heterogeneous attenuation (white arrow) and corresponding to diffusely heterogeneous parenchymal echotexture on ultrasound images (C).

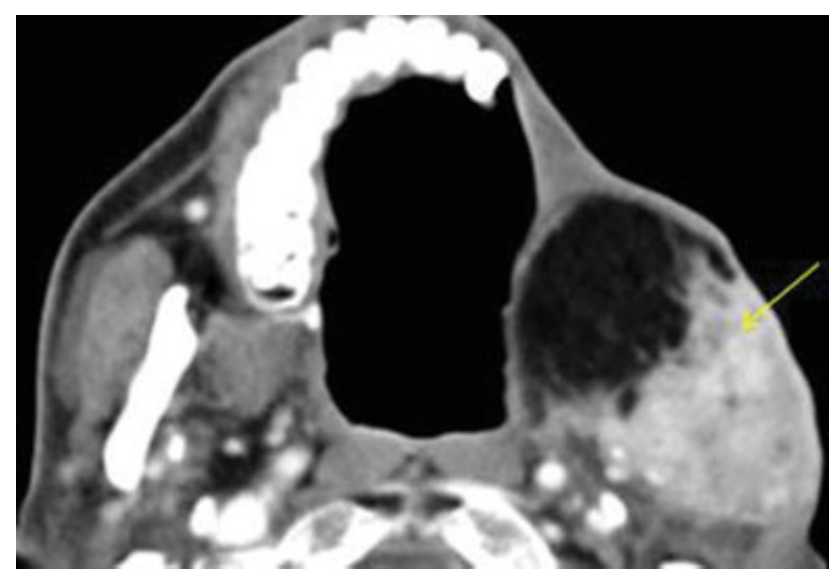

Fig. 19 Tumor recurrence at flap margin. Expansile enhancing soft tissue density mass (yellow arrow) at the margin of the flap in a 69year-old treated for carcinoma left upper alveolus suggestive of tumor recurrence.

carcinoma of temporal bone, or external auditory canal and malignant peripheral nerve sheath tumors ${ }^{19}$ (- Fig. 17). Thyroid malignancies developing postradiation can pose a challenge in establishing a causal effect due to its inherently high prevalence. Some cases of thyroiditis, however, are reported in literature more commonly occurring than thyroid malignancies, whereby there is heterogeneous replacement of normal thyroid parenchyma, with the gland eventually undergoing atrophy and patients developing hypothyroidism (-Fig. 18).

\section{Recurrence after Radiation Therapy}

The development of a new expansile enhancing mass or new area of FDG avidity (-Fig. 19) at the tumor bed or surgical flap margins, lymphadenopathy (-Fig. 20), or evidence of new bone or cartilage destruction ( - Fig. 21) must be viewed

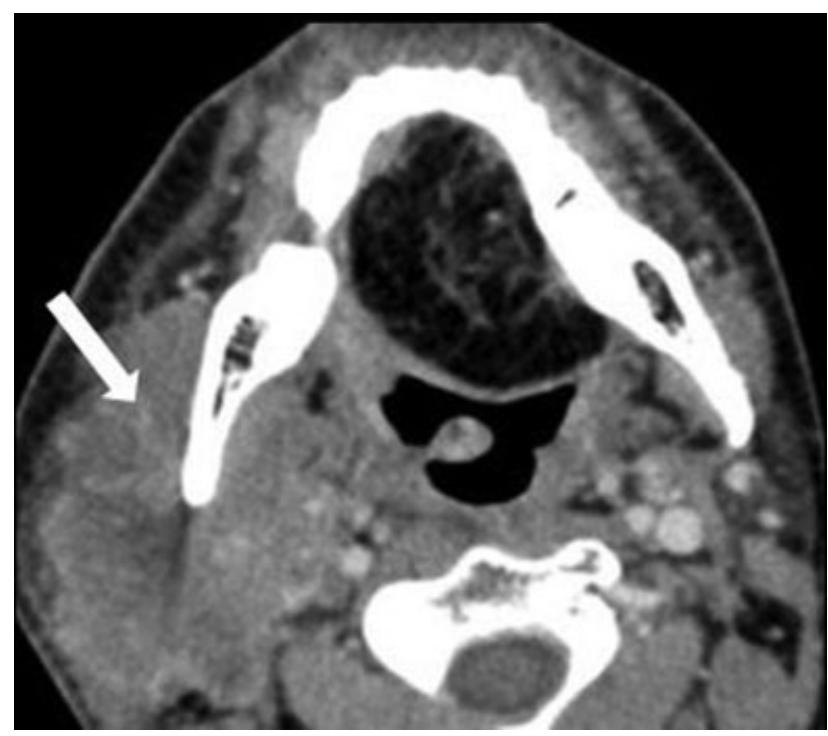

Fig. 20 Recurrence in node. Right cervical nodal recurrence (thick white arrow) in a 51-year-old man with carcinoma left lateral border of the tongue, post-operative and post-adjuvant chemoradiotherapy.

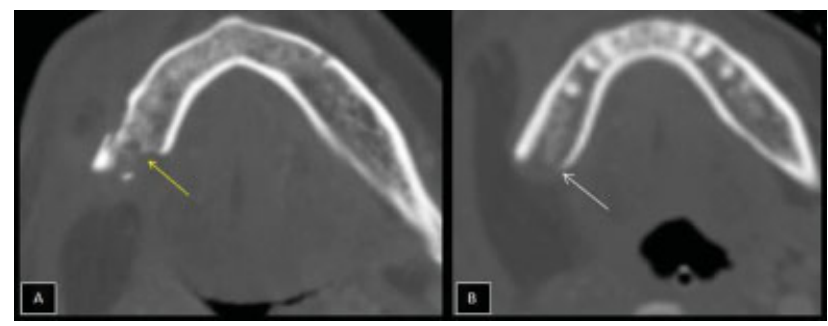

Fig. 21 (A, B) Recurrence with bone destruction. New bone destruction (yellow arrow) at the site of previous sharp surgical margins (white arrow) in right hemimandible 2 years after treatment completion suggestive of tumor recurrence.

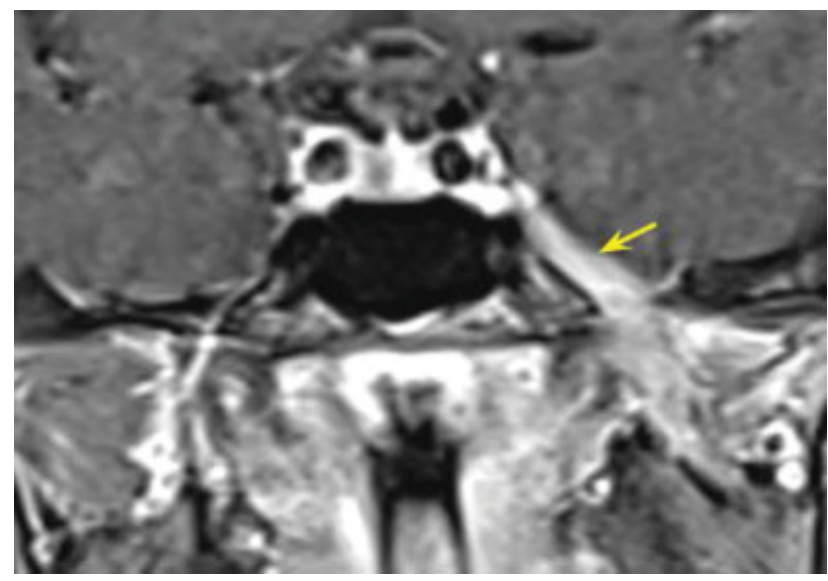

Fig. 22 Perineural spread. Contrast-enhanced fat saturated T1weighted coronal MR image shows thickening and enhancement (yellow arrow) along the mandibular division of trigeminal nerve (V3) in the left foramen ovale in a 66-year-old woman with squamous cell carcinoma of the left maxillary sinus, 14 months after treatment completion. MR, magnetic resonance imaging.

with suspicion for recurrence. Recurrence can also occur uniquely in the head and neck region in the form of perineural spread (- Fig. 22). 


\section{Conclusion}

RT continues to remain the workhorse in the treatment of head and neck carcinomas. Even with the newer RT techniques that are aimed at reducing therapy-related morbidities, some effects are inevitable. The most common and important findings encountered in the treated head and neck include changes in the mucosa, soft tissues, glands; changes in bony and cartilaginous structures; damage to nervous systems; and vascular systems and radiation-induced neoplasms. Many of these findings may be initially identified at imaging alone and therefore, familiarity with these spectrums of changes is important and so is differentiating them for tumor recurrence.

Conflicting Interest

None declared.

\section{Acknowledgments}

The authors would like to thank Drs. Aditi Chandra and Dayananda Lingegowda for their contribution in design and conception. The authors would like to thank Drs. Priya Ghosh, Argha Chatterjee, and Bharat Gupta for reviewing the manuscript and for adding to the intellectual content.

\section{References}

1 Saito N, Nadgir RN, Nakahira M, et al. Posttreatment CT and MR imaging in head and neck cancer: what the radiologist needs to know. Radiographics 2012;32(05):1261-1282, discussion 12821284

2 Peter M. Som, Eric M. Genden. The Posttreatment Neck: Clinical and Imaging Considerations. Peter M. Som and Hugh D. Curtin editors. Head and Neck Imaging. 2011. Mosby Elsevier. 5th edition. Volume 2; 2249-2250

3 Kim J, Shin ES, Kim JE, Yoon SP, Kim YS. Neck muscle atrophy and soft-tissue fibrosis after neck dissection and postoperative radiotherapy for oral cancer. Radiat Oncol J 2015;33(04):344-349

4 Benveniste MF, Gomez D, Carter BW, et al. Recognizing radiation therapy-related complications in the chest. Radiographics 2019; 39(02):344-366
5 Alhilali L, Reynolds AR, Fakhran S. Osteoradionecrosis after radiation therapy for head and neck cancer: differentiation from recurrent disease with CT and PET/CT imaging. AJNR Am J Neuroradiol 2014;35(07):1405-1411

6 Chrcanovic BR, Reher P, Sousa AA, Harris M. Osteoradionecrosis of the jaws-a current overview-part 1: physiopathology and risk and predisposing factors. Oral Maxillofac Surg 2010;14(01):3-16

7 Glastonbury CM, Parker EE, Hoang JK. The postradiation neck: evaluating response to treatment and recognizing complications. AJR Am J Roentgenol 2010;195(02):W164-71

8 Dassarath M, Yin Z, Chen J, Liu H, Yang K, Wu G. Temporal lobe necrosis: a dwindling entity in a patient with nasopharyngeal cancer after radiation therapy. Head Neck Oncol 2011;3:8

9 Wang Y-XJ, King AD, Zhou H, et al. Evolution of radiation-induced brain injury: MR imaging-based study. Radiology 2010;254(01): 210-218

10 Shah R, Vattoth S, Jacob R, et al. Radiation necrosis in the brain: imaging features and differentiation from tumor recurrence. Radiographics 2012;32(05):1343-1359

11 Zhao F, Bu L, Zhang W, et al. Herpes simplex encephalitis during radiation therapy in a patient with nasopharyngeal carcinoma. Transl Cancer Res 2017;6(06):1149-1155

12 Sermer DJ, Woodley JL, Thomas CA, Hedlund JA. Herpes simplex encephalitis as a complication of whole-brain radiotherapy: a case report and review of the literature. Case Rep Oncol 2014;7(03): 774-779

13 Dropcho EJ. Neurotoxicity of radiation therapy. Neurol Clin 2010; 28(01):217-234

14 Warade AC, Jha AK, Pattankar S, Desai K. Radiation-induced brachial plexus neuropathy: A review. Neurol India 2019;67 (suppl):S47-S52

15 Wilbers J, Kappelle AC, Kessels RP, et al. Long term cerebral and vascular complications after irradiation of the neck in head and neck cancer patients: a prospective cohort study: study rationale and protocol. BMC Neurol 2014;14:132

16 Suárez C, Fernández-Alvarez V, Hamoir M, et al. Carotid blowout syndrome: modern trends in management. Cancer Manag Res 2018; 10:5617-5628

17 Choi HS, Jeong BK, Jeong $\mathrm{H}$, et al. Carotid sparing intensity modulated radiotherapy on early glottic cancer: preliminary study. Radiat Oncol J 2016;34(01):26-33

18 Gujral DM, Shah BN, Chahal NS, Senior R, Harrington KJ, Nutting $\mathrm{CM}$. Clinical features of radiation-induced carotid atherosclerosis. Clin Oncol (R Coll Radiol) 2014;26(02):94-102

19 Thiagarajan A, Iyer NG. Radiation-induced sarcomas of the head and neck. World J Clin Oncol 2014;5(05):973-981 